\section{La evaluación multidisciplinaria en disforia de género: reporte de caso y revisión de la literatura}

Cynthia Jiménez Zarazúa', Magdalena Rodríguez Salinas ${ }^{1}$, Karla Motilla Negrete ${ }^{1}$, Jorge Mascareñas Ruíz ${ }^{1}$

1 Departamento de Psiquiatría, Hospital Universitario, Universidad Autónoma de Nuevo Leon, Monterrey, Mexico

\section{Resumen}

El Trastorno de Identidad de Género (TIG), Transexualismo (T) o Disforia de Género (DG), se caracteriza por la experiencia subjetiva de pertenecer al sexo opuesto y la presencia de sentimientos de malestar o desacuerdo por el sexo anatómico. Esta discrepancia suele implicar cambios físicos y sociales importantes, así como riesgo de presentar comorbilidad psiquiátrica asociada. Su prevalencia es poco frecuente y se desconoce su etiopatogenia. Las guías internacionales para la atención de estos pacientes recomiendan una evaluación desde equipos multidisciplinarios para realizar un diagnóstico correcto y ofrecer un tratamiento con el objetivo principal de lograr un equilibrio perdurable, una comodidad personal con el género con el que se identifica y la realización total del paciente. La intención de este trabajo es presentar nuestra experiencia en un caso de DG/T/TIG evaluado por un equipo de profesionales de la salud.

Palabras clave: personas transgenero, identidad de género, identificación, ajuste social, psiquiatría.

\section{The multidisciplinary evaluation on gender dysphoria: a case report and review of the literature}

\section{Abstract}

Gender Identity Disorder (GID), Transsexualism (T) or Gender Dysphoria $(D G)$, is characterized by the subjective experience of belonging

\section{Correspondencia:}

”cyn.jz@hotmail.com 
to the opposite sex and the presence of feelings of discomfort or disagreement with the own anatomical sex. This discrepancy often involves significant physical and social changes such an elevated risk of psychiatric comorbidity. Its prevalence is low and the pathogenesis is unknown. International guidelines for care of these patients recommend a multidisciplinary evaluation to get a correct diagnosis and provide treatment with the main objective to achieve a long-lasting personal comfort with the gender the patient identifies with and total fulfillment of themselves. The aim of this paper is to present our experience with a case of GID/T/DG evaluated by a team of health care professionals.

Key words: transsexualism, transgendered persons, gender identity, identification, social adjustment, adult.

\section{Introducción}

Harry Benjamín, endocrinólogo y sexólogo en una conferencia pronunciada en Junio de 1976 en Nueva York, afirmó:

"Me gustaría recordar a todos un hecho importante y fundamental:

La diferencia entre sexo y género. Sexo es lo que se ve. Género es lo que se siente.

La armonía entre ambos es esencial para la felicidad humana."

Las tendencias sexuales de hombres y mujeres fueron consideradas por las distintas ramas de la medicina y la sexología del siglo XIX, como estrictamente ligadas al sexo anatómico, cualquier variante a esa tendencia era considerada patología. No obstante, los primeros estudios antropológicos y sociológicos contrastan con la afirmación inicial de que el sexo anatómico debía corresponder a una conducta sexual exclusiva y excluyente [1].

Cauldwell en 1949, fue el primero en usar el término Transexualismo; término que fue popularizado por Harry Benjamin en 1966 [2, 3]. El término Disforia de género fue introducido por Norman Fisk en 1973 en el sentido de que el trastorno produce ansiedad asociada al conflicto entre la identidad sexual y el sexo anatómico [4].

En la década de los sesenta y setenta se creía que la identidad sexual en la niñez estaba tan poco diferenciada, que era posible a través del ambiente criar a un varón genético como niña. Las consecuencias devastadoras presentadas en el caso de David Reimer son un ejemplo en la literatura de que la identidad de género programada es irreversible y no depende de factores externos [5-7]. 
A lo largo de las últimas décadas, algunos estudios han intentado encontrar relación entre las variantes de la expresión del género con aspectos genéticos, familiares, neuroendocrinos y sociales [8-10]. La tendencia actual es integrar de manera flexible la presentación de los distintos aspectos que conforman el género en todos los niveles.

\section{Caso clínico}

Algunos datos presentados se han modificado por razones éticas. La evaluación del paciente fue integral, participaron médicos de psiquiatría, psicología, ginecología y endocrinología.

Femenino de 20 años de edad, soltera, católica, preparatoria trunca, desempleada. No presentó complicaciones perinatales, tuvo un buen desarroIlo psicomotor, sin antecedentes médicos relevantes. Niega antecedentes de abuso físico, sexual o psicológico. Acude al servicio de Psiquiatría para "definir su orientación sexual", lo que hace para complacer a sus padres, sin embargo la paciente realmente buscaba información y orientación para una reasignación de sexo. A partir de aquí nos referiremos al paciente de acuerdo al género con el que se identifica.

El paciente refirió que desde muy pequeño se ha sentido diferente al resto de las niñas, con predilección a convivir con niños y participar en juegos de varones. Recuerda dificultad para adaptarse a actividades femeninas, describe que fue en la infancia temprana cuando se enfrentó con su género de nacimiento: "Caí en cuenta de que yo era una niña y mis sueños no se cumplirían"; ya que en su etapa escolar él pensaba sus planes a futuro en un rol masculino: "Algún día seré padre de familia, trabajaré como mi papá y cuidaré a mi esposa e hijos". Vivía gran conflicto por expresarse del modo en que se sentía por temor a perder a su familia, y relata que inicia una constante sensación de insatisfacción, frustración y ansiedad.

En la adolescencia el desarrollo del cuerpo femenino que rechazaba fue descrito como traumático, sobre todo la menarquia. Comenzó a utilizar vendajes para encubrir el volumen mamario. A los 14 años inicia con fantasías sexuales, afirmó haber sentido atracción por las mujeres desde siempre, pero es hasta esta edad en que se vuelve algo totalmente consciente llegando a fantasear y tener sueños de contenido erótico con mujeres. Su orientación sexual es definida como heterosexual al describirse: "Soy un hombre heterosexual encerrado en el cuerpo de una mujer". Niega inicio de vida sexual y relaciones de pareja. Niega masturbación. A los 17 años, posterior a comunicar su situación con sus amigos cercanos corta su cabello y comienza a vestir varonilmente, además pidió ser llamado por el diminutivo de su nombre, ya que es más masculino.

Desde hace 2 años presenta síntomas recurrentes de tristeza, sentimientos de minusvalía, insatisfacción e irritabilidad; sintomatología que se exacerbó en el último mes antes de la evaluación con presencia de llanto fácil, tristeza continua, sentimientos de culpa, anhedonia, insomnio mixto e hiperfagia; además de presencia de ataques de pánico intermitentes con agorafobia; sintomatología que repercute negativamente en la esfera social, laboral o en otras áreas vitales del paciente.

\section{Examen mental, exploración física y clinimetría}

Paciente de edad aparente igual a la cronológica, con vestimenta y manierismos varoniles, pelo corto, maquillaje nulo. Orientado en tiempo, lugar y persona. Afecto ansioso, humor acorde. Niega alteraciones sensoperceptivas. El contenido de su pensamiento es de culpa y minusvalía. Presenta insomnio mixto, hiperfagia y disminución de la libido. De inteligencia promedio por clínica, juicio preser- 
vado, niega ideas de muerte, ideación o planeación suicida u homicida.

Exploración física y neurológica: IMC 25.5 (sobrepeso), resto sin datos de relevancia clínica. Sin alteraciones en la exploración neurológica. Estudios de laboratorio básicos normales. Las evaluaciones ginecológica y endocrinológica se reportaron normales.

Escala de depresión de Hamilton: 21 puntos (1829 depresión mayor); Inventario de depresión de Beck: 25 (>15 depresión grave); Escala de ansiedad de Hamilton: 33 puntos (>15 ansiedad mayor).

\section{Perspectiva psicodinámica. Análisis estructural}

Dentro de una evaluación estructural de la personalidad se evalúa principalmente el contacto con la realidad, su identidad y los mecanismos de defensa que utiliza una persona.

Criterio de realidad preservado, conservando un adecuado contacto y relación con la realidad, refiere: "Sé que nací como una mujer, sé que mi cuerpo es de una mujer, pero yo no me siento así". Bajo condiciones estresantes puede alterarse la percepción subjetiva de su entorno como cuando comenta: "Me siento observada todo el tiempo, aunque sé que no hay nadie ahí".

Tiene claro quién es, y logra apreciar una imagen completa de los demás, tolera la ausencia física del otro sin que esto le provoque ansiedad o frustración, tiene amistades duraderas, profundas y constantes.

Sus principales mecanismos de defensa se clasifican como represivos o inhibitorios, llevándolo a presentar síntomas fóbicos. Significa también, que en ocasiones llega a contener dentro de sí mismo sentimientos negativos como enojo o frustración y puede fallar en reconocer algunas emociones como propias. Tiene capacidad para posponer las satis- facciones. Sus defensas de carácter tienden a llevarlo a tener actitudes de dependencia. Cuando comete una equivocación, es capaz de experimentar culpa y reparar sus acciones.

\section{Pruebas psicológicas}

Las pruebas aplicadas fueron: Escala Wechsler de Inteligencia para Adultos WAIS-III, pruebas proyectivas gráficas, Psicodiagnóstico de Rorschach y Test de Apercepción Temática TAT. Se solicitaron para valorar funcionamiento cognitivo y determinar su estilo de pensamiento.

El resultado total de la escala WAIS-III fue 103 puntos, que corresponde a nivel intelectual normal promedio. En las pruebas gráficas y TAT se identificó con el sexo masculino, al dibujarse a sí mismo en rol de varón y representar dificultades cotidianas con personajes masculinos. El test de Rorschach reveló una puntuación del Índice de Trastorno de Percepción-Pensamiento de 2, lo cual significa que posee habilidad para pensar claramente y percibir de manera adecuada. Obtuvo 6 puntos en el Índice de Suicidio (8 puntos significa riesgo inminente de autoagresión) lo cual señala un riesgo importante, así como 3 variables en el límite de referencia a aspectos depresivos y un auto concepto devaluado.

Los datos obtenidos con las pruebas no contraindican el proceso de reasignación de sexo, sin embargo recomiendan tomar en cuenta la predisposición a la depresión y el riesgo de autoagresión que pudiera presentarse en un momento de crisis.

\section{Evaluación de familia}

Se trata de una familia nuclear tradicional, en etapa de crianza de los hijos. Conformada por ambos padres y tres hijas, siendo el paciente identificado el menor de ellos. En la evaluación realizada en cinco sesiones se observó un sistema de valores interno rígido, con expectativas inmodificables del "deber ser". Esta característica de la familia dificultó 
la apertura al concepto de transexualismo, rechazándolo desde lo cultural, religioso y social. Dentro de la familia hay límites poco claros, manifiesto en el sobre involucramiento de los padres en el cuidado de los hijos. Lo cual se complementa con las defensas de carácter dependientes observados en el paciente.

La red de apoyo en los pacientes transgenero es vital, sin embargo no es raro que la familia no sea el apoyo primario, sino la familia extensa $u$ otras redes (amigos, maestros, instituciones, grupos de apoyo, médicos y/o terapeutas) los que ejercen este papel. En este caso solo 1 miembro del subsistema filial mostró apertura al proceso de reasignación de sexo.

\section{Diagnóstico DSM-5 y tratamiento}

- Disforia de Género en el adulto.

- Trastorno depresivo mayor, recidivante, moderado.

- Trastorno de angustia con agorafobia.

De acuerdo a los criterios diagnósticos del DSM-5 [11] el paciente presenta desde la edad escolar una marcada incongruencia entre el sexo que siente y expresa con respecto al sexo asignado, esto es de manera continua y permanente durante la pubertad, adolescencia y al momento de la evaluación. Además de fuerte rechazo de sus caracteres sexuales y genitales femeninos; fuerte deseo de pertenecer al otro sexo y ser tratado como hombre, lo que es congruente con la sensación de pertenencia al sexo masculino. Esto se acompaña de un malestar clínicamente significativo. Se descartaron otras condiciones como: trastornos del desarrollo psicosexual, enfermedad orgánica, otras enfermedades psiquiátricas como trastornos psicóticos, trastorno obsesivo compulsivo o trastorno grave de la personalidad. Tampoco se encontraron dificultades cognitivas que pudieran afectar el juicio y la toma de decisiones sobre el propio cuerpo y la salud.
Se ofreció tratamiento farmacológico para las comorbilidades psiquiátricas con paroxetina $20 \mathrm{mg}$ cada 24 horas y alprazolam 0.25 mg cada 8 horas; psicoterapia individual con el principal objetivo de fortalecer las habilidades adaptativas y de resiliencia del paciente. Además de educación sobre los tratamiento disponibles de reasignación de sexo.

\section{Consideraciones}

El paciente durante la entrevista psiquiátrica negó ideación o planeación suicida; sin embargo en la evaluación de pruebas psicológicas el índice de suicidabilidad se reportó elevado. Hallazgo que deberá ser tomado en cuenta para el manejo de este paciente.

\section{Revisión del tema}

\section{Aspectos epidemiológicos}

El Trastorno de Identidad de Género (TIG), Transexualismo o Disforia de Género (DG) es poco frecuente en todo el mundo. La prevalencia de vida varía de $0.001 \%$ -

0.002\%29 a 0,0019\%-0,0024\% [12]. En estudios epidemiológicos anteriores, como el realizado por Landén y colaboradores se hizo una revisión de estudios epidemiológicos realizados desde los años 60 hasta el año 1992, se reportó una relación del tipo mujer a hombre $(\mathrm{MaH})$ al tipo de hombre a mujer (HaM) de 3 a $5[29,30]$. Por otra parte, de acuerdo a estudios epidemiológicos más recientes, la prevalencia de los tipos de HaM y MaH se ha estimado en 1: 12.900 y 1: 33.800 respectivamente, y las relaciones entre el tipo $\mathrm{HaM}$ a $\mathrm{MaH}$ han sido reportados como 2.43:1 [13]. Este incremento de la prevalencia se atribuye a una mayor

aceptación por parte de la sociedad, lo que ha aumentado la búsqueda de tratamientos de reasignación de sexo [14]. 


\section{Etiopatogenia}

Se desconocen las causas. Anteriormente las teorías psicosociales que eran más aceptadas para explicar las conductas sexuales de los seres humanos, se basaban en factores no cuantificables y subjetivos, dando un peso importante al ambiente social como determinante de la conducta sexual [10]; proponían que la identidad de género y todas las características de la sexualidad estaban sujetas a entrenamiento por parte de las personas que nos rodean [15-17], dichas teorías actualmente han sido superadas por las biológicas.

Las teorías biológicas actuales sugieren anomalías en el desarrollo temprano del cerebro por influencia de esteroides sexuales [18-20]. Estudios neurobiológicos [21-23] han propuesto como algunos de los causantes al estrés materno, la exposición a fármacos durante el embarazo y otros padecimientos como la hiperplasia adrenal congénita, síndrome de insensibilidad a los andrógenos y la deficiencia de 15-alfa-reductasa [24,25].

Se ha estudiado la participación de factores genéticos en casos de TIG/T/DG con reportes de casos de gemelos y hermanos no gemelos, así como en reportes de casos donde más de un miembro de una familia es TIG/T/DG [26]. También se han descrito polimorfismos de receptores a esteroides y andrógenos, así como patrones de heredabilidad de la orientación sexual [27-29]. Sin embargo no existe información disponible de variantes específicas de un gen que potencien o reduzcan la probabilidad de desarrollar TIG/T/DG .

\section{Diagnóstico}

El TIG/T/DG se caracteriza por la experiencia subjetiva de la propia individualidad como perteneciente al sexo opuesto y la presencia de sentimientos de malestar o desacuerdo por el sexo anatómico. En el Manual Diagnóstico y Estadístico de la Asociación Psiquiátrica Americana en su cuarta y quinta edición (DSM-IV-TR, DSM V), así como en la décima edición de la Clasificación Estadística Internacional de Enfermedades (CIE-10) se incluyen descripciones de trastorno de identidad de género o disforia de género en la infancia y la edad adulta [11, 30, 31]. (Tabla 1)

\section{Comorbilidades psiquiátricas}

Se ha demostrado que la presencia de comorbilidad de trastornos psiquiátricos influye en la evolución y en el pronóstico de TIG/T/DG [32,33]; por lo que es de suma importancia la evaluación de comorbilidades psiquiátricas en este grupo de pacientes. Existen varios estudios al respecto, sin embargo los hallazgos son inconsistentes. Aunque algunos estudios indican que la prevalencia de trastornos psiquiátricos en este grupo de pacientes es similar a los controles [34]; otros estudios demostraron que la TIG/T/DG se asocia con una mayor prevalencia de trastornos psiquiátricos y un mayor índice de suicidios en comparación con la población general [35-38]. Además, varios estudios han demostrado que las comorbilidades psiquiátricas varían dependiendo del sexo anatómico, siendo la tasa de $\mathrm{MaH}$ menor que la de HaM $[39,40]$. Las psicopatologías más frecuentemente encontradas incluyen los trastornos depresivos, ansiedad, toxicomanías y trastornos de la personalidad, principalmente trastorno por dependencia, narcisista y límite [32-40].

Existe poca información disponible sobre las conductas suicidas de los pacientes con TIG/T/DG [41]. En estudios anteriores se reportan tasas de intentos de suicidio del 19 al 25\% [42] y en encuestas no aleatorias más recientes de pacientes con TIG/T/DG se encontró que hasta un tercio de los encuestados reportó haber cometido uno o más intentos de suicidio durante su vida [38, 43-45]. Estos parecen ocurrir con más frecuencia entre adolescentes y adultos jóvenes [46]. Como factores asociados se han identificados altos índices de depresión, ansiedad y abuso de sustancias [41, 43]. 
Tabla 1. Clasificaciones diagnósticas para adultos. CIE-10, DSM-IV TR y DSM-5.

\begin{tabular}{|c|c|}
\hline $\begin{array}{l}\text { Transexualismo } \\
\text { CIE-10 }\end{array}$ & $\begin{array}{l}\text { - Deseo de vivir y ser aceptado como miembro del sexo opuesto, acompañado de malestar o } \\
\text { desacuerdo con el sexo anatómico propio. Deseos de tratarse quirúrgica u hormonalmente } \\
\text { - Presente por lo menos dos años } \\
\text { - No es síntoma de otro trastorno mental, anomalía intersexual, genética o cromosomas } \\
\text { sexuales }\end{array}$ \\
\hline $\begin{array}{l}\text { Trastorno de la } \\
\text { identidad sexual } \\
\text { DSM-IV TR }\end{array}$ & $\begin{array}{l}\text { - Identificación acusada y persistente con el otro sexo (no solo el deseo de obtener las supuestas } \\
\text { ventajas relacionadas con las costumbres culturales). } \\
\text { - Malestar persistente con el propio sexo o sentimiento de inadecuación con su rol } \\
\text { - La alteración no coexiste con una enfermedad intersexual } \\
\text { - Provoca malestar clínicamente significativo o deterioro social, laboral o de otras áreas } \\
\text { - Codificar según la edad actual: En niños, adolescentes o adultos }\end{array}$ \\
\hline $\begin{array}{l}\text { Disforia de género } \\
\text { DSM-5 }\end{array}$ & $\begin{array}{l}\text { - Marcada incongruencia entre el sexo que uno siente o expresa y el asignado, de una duración } \\
\text { - Incongruencia percibida ante los caracteres sexuales propios } \\
\text { - Fuerte deseo por desprenderse de los caracteres sexuales propios } \\
\text { - Fuerte deseo de poseer los caracteres sexuales correspondientes al sexo opuesto } \\
\text { - Fuerte deseo de ser de otro sexo distinto al asignado } \\
\text { - Fuerte deseo de ser tratado como del otro sexo } \\
\text { - Fuerte convicción de que uno tiene los mismos sentimientos y reacciones típicos del otro } \\
\text { sexo. } \\
\text { - Asociado a malestar clínicamente significativo o deterioro social, laboral u otras áreas } \\
\text { importantes. } \\
\text { - Especificar si: } \\
\text { - Con trastorno de desarrollo sexual } \\
\text { - Postransición }\end{array}$ \\
\hline
\end{tabular}

\section{Generalidades sobre el tratamiento}

Anteriormente en los tratamientos de los pacientes con TIG/T/DG se buscaba un cambio físico al sexo deseado de la manera más completa posible y; aunque es cierto que muchas personas necesitan tanto la terapia hormonal y las cirugías para aliviar la disforia que esto les ocasiona [47], esto no es siempre es así, también hay otras personas que sólo necesitan una de estas opciones o ninguna de ellas [48]; por ejemplo, algunas personas alivian los síntomas de disforia con realizar cambios en el rol y la expresión de género sin la necesidad de terapia hormonal o cirugías; otros si necesitan feminizar o masculinizar su cuerpo para aliviar sus síntomas, sin embargo no necesitan cirugías; en cambio otros si se practican cirugía pero no terapia hormonal. Por lo que tratamiento de TIG/T/DG debe estar individualizado a las necesidades de cada persona.

Dado el espectro de variabilidad de género que puede existir, los profesionales de la salud pueden ayudar a las personas con TIG/T/DG a afirmar su identidad de género, explorar las diferentes alternativas para la expresión de esa identidad, y tomar decisiones sobre las opciones de tratamientos médicos para aliviar la disforia de género [47]. 
Tabla 2. Opciones de tratamiento médico y psicológico de la disforia de genero

\section{Cambios en la expresión y rol de género}

- Puede involucrar el vivir tiempo parcial o completo en otro rol de género, en consonancia con la identidad de género de la persona

\section{Terapia hormonal para feminizar o masculinizar el cuerpo}

\section{Cirugías para cambiar las características sexuales primarias y/o secundarias}

- Por ejemplo, senos/pechos, genitales externos y/o internos, rasgos faciales, contorno corporal

\section{Psicoterapia}

- Individual, de pareja, familiar o grupal

o Explorar la identidad, rol y expresión de género

o Abordar el impacto negativo de la disforia de género y el estigma en la salud mental

o Aliviar la transfobia internalizada

o Aumentar el apoyo social y entre pares

o Mejorar la imagen corporal

o Promover resistencia.

De acuerdo a las Normas de atención para la salud trans y con variabilidad de género de la Asociación Mundial para la Salud Transgenero, existe una variedad de opciones terapéuticas para las personas con TIG/T/DG que buscan atención médica [47] (Tabla 2).

\section{Pronóstico}

El pronóstico de las personas con TIG/T/DG es variable. Dependerá de las características individuales de cada paciente incluyendo edad, género asignado, orientación sexual y comorbilidades. La opción que el paciente elija como tratamiento influye también en el curso y pronóstico.

Varios estudios han reportado que la reasignación de sexo se asocia con cambios favorables no sólo en los síntomas disfóricos, si no en las distintas áreas de la vida del paciente como la vida familiar, social y las relaciones sexuales [49-51]. Sin embargo, la prevalencia de algunos trastornos psiquiátricos como depresión y trastornos de ansiedad continúa más alta que en la población general [35, 49].

En una revisión sistemática de 28 estudios que realizó Murad et al. para determinar los beneficios de la terapia hormonal, se encontraron mejorías en la disforia de género, el funcionamiento psicológico y comorbilidades, disminución de las tasas de suicidio, mayor satisfacción sexual y en general una mejor calidad de vida posterior al tratamiento, sin embargo no se encontró evidencia concluyente para considerar a la terapia hormonal como la única causa de estas mejorías.[52]

Algunos estudios reportan diferencias en el pronóstico dependiendo del sexo anatómico; sugieren que 
el grupo HaM puede tener peores resultados en la reasignación de sexo que sus contrapartes $\mathrm{MaH}$ [52]; lo que soporta a otros estudios que señalan que las personas transexuales de HaM presentan niveles más altos de comorbilidades psiquiátricas que la población general [53] y muestran menor nivel de funcionamiento psicológico que el grupo $\mathrm{MaH}$ [54].

También en el estudio de Murad et al., se observó que las personas que tienen un inicio más temprano de los síntomas y los que inician el tratamiento antes de la adultez presentan un mejor pronóstico [52].

En 2012 Michel et al., revisó la literatura disponible desde los años cincuenta a la fecha respecto a la reasignación quirúrgica de género. Una de las principales dificultades para el estudio sistematizado de estos casos es que con el paso de los años el término y los criterios diagnósticos para TIG/T/ DG han variado continuamente y se han realizado en poblaciones heterogéneas utilizando metodología diversa. Sin embargo, algunos resultados de esos estudios son reagrupables; como el grado de satisfacción posterior al procedimiento. El cambio se considera satisfactorio cuando el paciente se encuentra contento con la transformación quirúrgica y adaptado al nuevo rol. En contraste, un resultado insatisfactorio se considera cuando empeoran las condiciones psicológicas y sociales del paciente posterior al procedimiento, en comparación con la condición previa al tratamiento. En esta revisión las tasas de satisfacción varían entre el 87-93\% tanto en HaM como en MaH. [55]

Posterior al procedimiento quirurgico puede haber sensación subjetiva de instatisfacción que puede llevar al arrepentimiento, usualmente esta sensación es temporal y no requiere una intervención. Pfäfflin y Junge revisaron la literatura al respecto y reportan que la mayoría de las veces la insta- tisfacción inicial está relacionada al dolor postoperatorio, complicaciones quirurgicas, pérdida de la pareja, del empleo y problemas familiares. Reportan que el arrepentimiento a largo plazo es raro; 0.5 $11.2 \%$ en HaM y $1-1.5 \%$ de los casos $\mathrm{MaH}$. En una examinación más detallada describen algunos aspectos asociados al arrepentimiento, como un diangóstico incorrecto, ausencia de un periodo de prueba en la vida real del género deseado y protocolos quirúrgicos no adecuados [56].

\section{Conclusión}

Pese a que no existen suficientes estudios de seguimiento a largo plazo para las distintas opciones de tratamiento disponibles, tampoco existen razones empíricas que sugieran que una solicitud de reasignación de sexo deba ser sistemáticamente rechazada. Si se realiza una evaluación completa y se tiene el diagnóstico certero, realizar un plan de manejo y seguimiento adecuados para nuestro paciente le permitirá conocer todas las posibilidades de tratamiento que existen, incluyendo la posibilidad de detener su tratamiento en el punto que así lo decida. La pauta que guiará el tratamiento es llevar al paciente a que sienta satisfacción consigo mismo, y retome satisfactoriamente todas las áreas de su vida.

\section{Bibliografía}

1. Gastó Ferrer C. Transexualidad. Aspectos Históricos y Conceptuales. Cuadernos de Medicina Psicosomática y Medicina de Enlace. 2006; (78):13-20.

2. Bergero T, Cano G, Giraldo F, Esteva I, Ortega MV, Gómez M, Gorneman I. La transexualidad: asistencia multidisciplinar en el Sistema Público de Salud. Rev. Asoc. Esp. Neuropsiq 2004; (89): 9-20.

3. Benjamín H. The Transexual Phenomenon. Nueva York, The Julian Press. 1996

4. Chiland C. Cambiar de sexo, Madrid, Ed. Biblioteca Nueva, 1999. 
5. Bao AM, Swaab DF. Sexual differentiation of the human brain: Relation to gender identity, sexual orientation and neuropsychiatric disorders. Frontiers in neuroendocrinology. 2011; 32(2):214-26.

6. Money J. Ablatio penis: normal male infant sex-reassigned as a girl. Arch. Sex Behav. 1975; 4(1):65-71

7. Money J, Erhardt AA. Man and Woman, Boy and Girl: The Differentiation and Dimorphism of Gender Identity from Conception to Maturity. Johns Hopkins University Press. 1972.

8. Fernandez R, Esteva I. Gomez-Gil E, Rumbo T, Almaraz MC, Roda E, Haro-Mora JJ, Guillamon A, Pásaro E. Association study of ERß, AR, and CYP19A1 Genes and MtF Transsexualism. J Sex Med, doi: 2015. 10.1111/jsm.12673.

9. Junger J, Habel U, Brohr S, Neulen J, Neuschaefer-Rube C, et al. More than Just Two Sexes: The Neural Correlates of Voice Gender Perception in Gender Dysphoria. 2015. PLoS ONE 9(11): e111672. doi:10.1371/journal.pone.0111672.

10. Orozco G, Ostrosky-Solís F, Salin-Pascual RJ, Borja KC, Castillo G. Bases Biológicas de la Orientación Sexual: Un estudio de las Emociones en Transexuales. Rev Neuropsicología, Neuropsiquiatría y Neurociencias. 2009; 9(1):9-24

11. American Psychiatric Association. Diagnostic and statistical manual of mental disorders (5th ed.). Washington, DC. 2013.

12. Landén $M$, Wälinder J, Lundström $B$, Prevalence, incidence and sex ratio of transsexualism. Acta Psychiatr Scand, 1996; (93), 221-223.

13. De Cuypere G, Van Hemelrijck M, Michel A, Carael B, Heylens G, Rubens R, Monstrey S. Prevalence and demography of transsexualism in Belgium. European Psychiatry, 2007; 22(3), 137-141.

14. Gil EG, Nogués JP, Perpiñá SA, de Pablo Rabassó J. Trastorno de la identidad sexual: Aspectos epidemiológicos, sociodemográficos, psiquiátricos y evolutivos. Cuadernos de medicina psicosomática y psiquiatria de enlace, 2001; (58), 76-83.

15. Crooks, R., Baur, K. Our sexuality. 1999; 7a. ed. USA: Brookx/ Cole Publishing Company.

16. Patterson CJ. Children of lesbian and gay parents. Child Development. 1999; 63(5): 1025-1042.

17. Tasker F. Lesbian mothers, gay fathers, and their children: A review. J Development Behavior and Pediatry. 2005; 26(3): 224240.

18. Dörner G, Poppe I, Stahl F, Kölzsch J, Uebelhack R. Geneand environment- dependent neuroendocrine etiogenesis of homosexuality and transsexualism. Exp. Clin. Endocrinol. 1991; 98: 141-150.

19. Bosinski HA, Peter M, Bonatz G, Arndt R, Heidenreich M, Sippell WG, Wille R. A higher rate of hyperandrogenic disorders in female-to-male transsexuals. Psychoneuroendocrinology. 1997; 22:361-380

20. van Goozen SH, Slabbekoorn D, Gooren LJ, Sanders G, CohenKettenis PT. Organizing and activating effects of sex hormones in homosexual transsexuals. Behav. Neurosci. 2002; 116, 982988.

21. Zhou JN, Hofman MA, Gooren LJ, Swaab DF. A sex difference in the human brain and its relation to transsexuality. Nature. 1995; $378,68-70$.
22. Kruijver FP, Zhou JN, Pool CW, Hofman MA, Gooren LJ, Swaab DF. Male-to- female transsexuals have female neuron numbers in a limbic nucleus. J. Clin. Endocrinol. Metab. 2000; 85, 20342041.

23. Chung WCJ, De Vries GJ, Swaab DF. Sexual differentiation of the bed nucleus of the stria terminalis in humans may extend into adulthood. The Journal of Neuroscience. 2002; 22(3), 10271033.

24. Camperio-Ciani A, Corna F, Capiluppi C. Evidence for maternally inherited factors favouring male homosexuality and promoting female fecundity. Proceedings of the Royal Society B: Biological Sciences. 2004; 271, 2217-2221.

25. Gooren L. The biology of human psychosexual differentiation. Hormones and Behavior. 2006; 50(4), 589-560.

26. Green R. Family cooccurrence of 'gender dysphoria': ten sibling or parent-child pairs. Arch. Sex. Behav. 2000; 29, 499-507.

27. Hamer DH, Hu S, Magnusos VL, Hu N, Pattatuci AM. A linkage between DNA markers on the $X$ chromosome and masculine sexual orientation. Science. 1993; 261(5119), 321-327.

28. Hu S, Pattatuci AM, Patterson C, Li L, Fulker DW, Cherny SS, et al. Linkage between sexual orientations and chromosome Xq28 in males but not in females. Nature Genetics. 1995; 11(3), 248256.

29. Bentz K, Hefler LA, Kaufmann L, Huber JC, Kolbus A, Tempfer CB. A polymorphism of the CYP17 gene related to sex steroid metabolism is associated with female-to-male but not male-tofemale transsexualism. Fertility and Sterility. 2008; 90(1), 56-59.

30. Organización Mundial de la Salud. CIE 10. Décima Revisión de la Clasificación Internacional de Las Enfermedades. Trastornos Mentales y del Comportamiento: Descripciones Clínicas y pautas para el Diagnóstico. Madrid: Meditor; 1992.

31. American Psychiatric Association. Diagnostic and Statistical Manual of Mental Disorders (Revised 4th ed.). Washington, DC. 2000.

32. Bodlund O, Kullgren G. Transsexualism—general outcome and prognostic factors: A five-year follow-up study of nineteen transsexuals in the process of changing sex. Archives of Sexual Behavior, 1996; 25(3), 303-316.

33. Michel A, Ansseau M, Legros JJ, Pitchot W, \& Mormont C. The transsexual: what about the future?. European Psychiatry, 2002; 17(6), 353-362.

34. Haraldsen IR, Dahl AA. Symptom profiles of gender dysphoric patients of transsexual type compared to patients with personality disorders and healthy adults. Acta Psychiatrica Scandinavica, 2000; 102(4), 276-281.

35. Hepp U, Kraemer B, Schnyder U, Miller N, Delsignore A. Psychiatric comorbidity in gender identity disorder. Journal of Psychosomatic research, 2005; 58(3), 259-261.

36. Bodlund O, Kullgren G, Sundbom E, Höjerback T. Personality traits and disorders among transsexuals. Acta Psychiatrica Scandinavica, 1993; 88(5), 322-327.

37. Cole CM, O'Boyle M, Emory LE, Meyer III WJ. Comorbidity of gender dysphoria and other major psychiatric diagnoses. Archives of sexual behavior, 1997; 26(1), 13-26.

38. Clements-Nolle K, Marx R, Katz M. Attempted suicide among transgender persons: The influence of gender-based 
discrimination and victimization. Journal of homosexuality, 2006; 51(3), 53-69.

39. Michel A, Mormont C, Legros JJ. A psycho-endocrinological overview of transsexualism. European Journal of Endocrinology, 2001; 145(4), 365-376

40. Gómez-Gil E, Trilla A, Salamero M, Godás T, Valdés M. Sociodemographic, clinical, and psychiatric characteristics of transsexuals from Spain. Archives of Sexual Behavior, 2009; 38(3), 378-392.

41. Mathy RM. Transgender identity and suicidality in a nonclinical sample: Sexual orientation, psychiatric history, and compulsive behaviors. Journal of Psychology \& Human Sexuality, 2003; 14(4), 47-65.

42. Dixen JM, Maddever H, Van Maasdam , Edwards PW. Psychosocial characteristics of applicants evaluated for surgical gender reassignment. Archives of Sexual Behavior, 1984; 13(3), 269-276.

43. Clements-Nolle K, Marx R, Guzman R, Katz M. HIV prevalence, risk behaviors, health care use, and mental health status of transgender persons: Implications for public health intervention. American Journal of Public Health, 2001; 91(6), 915

44. Grossman AH, D'Augelli AR. Transgender Youth and LifeThreatening Behaviors. Suicide and Life-Threatening Behavior, 2007; 37(5), 527-537.

45. Kenagy GP. Transgender health: Findings from two needs assessment studies in Philadelphia. Health \& social work, 2005; 30(1), 19-26

46. Bradford J, Reisner SL, Honnold JA, Xavier J. Experiences of transgender-related discrimination and implications for health: Results from the Virginia Transgender Health Initiative Study. American journal of public health, 2013; 103(10), 1820-1829.

47. Coleman E., et al. Standards of Care for the health of transexual, transgender and gender-nonconforming people, versión 7. International Journal of Transgenderism, 2012; 13(4), 165-232.

48. Bockting, W. O. Psychotherapy and the real-life experience: From gender dichotomy to gender diversity. Sexologies, 2008; 17(4), 211-224.

49. DeCuypere G, Elaut E, Heylens G, et al. Long-term followup: psychosocial outcome of Belgian transsexuals after sex reassignment surgery. Sexologies. 2006; 15, 126-133.

50. DeCuypere G, Sjoen GT, Beerten R, et al. Sexual and physical health after sex reassignment surgery. Archives of Sexual Behavior. 2005; 34, 679-690.

51. Kuiper B, Cohen-Kettenis P. Sex reassignment surgery: a study of 141 Dutch transsexuals. Archives of Sexual Behavior. 1998; 17, 439-457.

52. Murad MH, Elamin MB, Garcia MZ, Mullan RJ, Murad A, Erwin PJ, Montori VM. Hormonal therapy and sex reassignment: a systematic review and meta-analysis of quality of life and psychosocial outcomes. Clinical Endocrinology, 2010; 72(2), 214-231.

53. Heylens G, Elaut E, Kreukels BPC, Paap MCS, Cerwenka S, Richter-Appelt $\mathrm{H}$, et al. Psychiatric characteristics in transexual individuals: multicentre study in four European countries. $\mathrm{Br} J$ Psychiatry, 2013:1-6, http://dx.doi.org/10.1192/bjp. bp.112.121954.

54. Prunas A, Vitelli R, Agnello F, Curti E, Fazzari P, Giannini F, Bini M. Defensive functioning in MtF and FtM transsexuals. Comprehensive psychiatry. 2015; 55(4), 966-971.

55. Michel A, Ansseau M, Legros JJ, Pitchot W, Mormont C. The transsexual: what about the future? Eur Psychiatry 2002; 17: 353-62.

56. Pfäfflin F. Regrets after sex reassignment surgery. In: Bockting WO, Coleman E, editors. Gender dysphoria, interdisciplinary approaches in clinical management. Binghamton, NY: The Haworth Press; 1992.

\section{Opina sobre este artículo:}
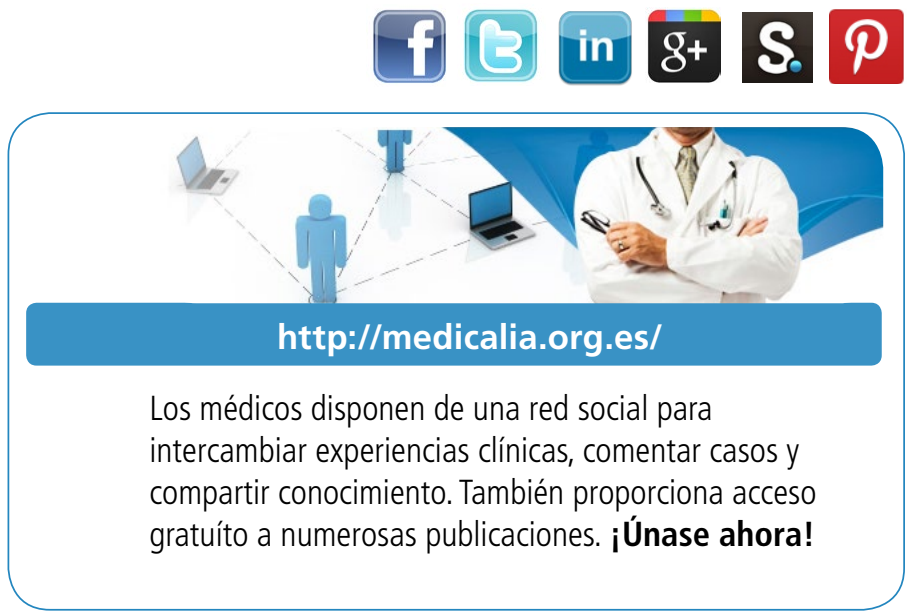

\section{Publique con iMedPub}

http://www.imed.pub

\section{Biomedicina}

$\checkmark$ Biomedicina es la primera revista de acceso abierto en Español, dedicada a todas las áreas de la medicina.

$\checkmark$ Recoge artículos de investigación sobre todas las áreas de la medicina. 